\title{
Cómo se Esfuerzan los Alumnos en Resolución de Problemas Matemáticos (I)
}

\section{Students' Effort in Mathematical Problem Solving (I)}

\author{
Joaquín Fernández Gago* \\ José Carrillo Yáñez ${ }^{* *}$
}

\begin{abstract}
Resumen
En este artículo tratamos cómo la forma en que los alumnos se esfuerzan influye a la hora de resolver problemas matemáticos. Para analizar cómo los alumnos se esfuerzan nos centramos en tres dimensiones: su atención, el lenguaje con ellos mismos o habla interior y cómo afrontan el reto, y para ello usamos un marco teórico que podría enmarcarse en las ideas de las habilidades ejecutivas como parte de la inteligencia. Lo hacemos con un estudio de casos de una alumna de $4^{\circ}$ de ESO, Sísifo. El estudio nos refleja que su forma de esforzarse está influyendo en sus fracasos a la hora de resolver problemas.
\end{abstract}

Palabras-clave: Esfuerzo en Resolución de Problemas. Estudio de Casos.

\begin{abstract}
In this paper we study how the way students make an effort influences problem solving. On analyzing students' effort, we focus on three dimensions: their attention, the language they use internally to talk with themselves and how they face the challenge; and that is the reason why we use a theoretical framework that includes executive abilities as part of intelligence. We choose a case study of a student in a Secondary school in the tenth year, Sísifo. The study brings to light the way effort influences problem solving.
\end{abstract}

Keywords: Effort in Problem Solving. Case Study.

\section{Introducción}

Autores como Schoenfeld (1992) y Vila y Callejo (2004) establecen la influencia de las creencias de los alumnos sobre las Matemáticas, sobre el aprendizaje de las Matemáticas, sobre la Enseñanza de las Matemáticas y sobre la Resolución de Problemas en la actuación de alumnos en resolución de problemas matemáticos. Schoenfeld (1985) establece, incluso, creencias y consecuencias como por ejemplo la creencia de que un problema de Matemáticas

\footnotetext{
* Doctor en Didáctica de las Matemáticas de la Universidad de Huelva (UHU), España. Profesor de Enseñanza Secundaria, de IES Miguel Romero Esteo de Málaga, Andalucía, España. Dirección Postal: Calle Ortiga $n^{\circ} 12$, 29190 Málaga, Andalucía, España. E-mail: joaquinf234@gmail.com

** Doctor en Filosofía y Ciencias de la Educación por Universidad de Sevilla (US), España. Catedrático de Universidad de Didáctica de la Matemática, Universidad de Huelva (UHU), Huelva, España. Dirección postal: Facultad de Ciencias de La Educación, Campus El Carmen, Avenida del 3 de Marzo s/n 21071 Huelva, España. E-mail: carrillo@uhu.es
} 
se resuelve en menos de 10 minutos, se deduce que si los alumnos no pueden resolver un problema en menos de 10 minutos abandonan.

En observaciones como profesor de Secundaria, algunas de las creencias anteriores explican las actitudes de los alumnos, pero no otras. Por ejemplo, imaginemos que un profesor de Matemáticas considera un objetivo importante la resolución de problemas con sus alumnos, considerándola tanto metodología como contenido. Supongamos, además, que propone un modelo con fases para enfrentarse a los problemas en los que se le recomiendan frases que el alumno se diga a sí mismo para mejorar, tanto en un problema, como en el procesos de mejora como resolutor a largo plazo. Hay alumnos que con la Resolución de Problemas crecen, se esfuerzan más y más a gusto, sintiéndose mejorar, pero otros puede que ni siquiera empiecen a esforzarse por mejorar en ese proceso a largo plazo, o la forma de esforzarse tradicional (asimilación y reproducción de algoritmos) la intensifica, llegando al fracaso al resolver problemas.

Lo anterior puede tener que ver con la formación de creencias sobre aspectos relacionados con el esfuerzo, o en la creencia de la posibilidad de cambio entre otros, que están incidiendo en la actuación del alumno. Nuestra experiencia como profesor de Matemáticas nos dice que, si pretendemos que la Resolución de Problemas sea tanto metodología como contenido, los aspectos anteriores están influyendo en los alumnos que sufren bloqueos, del que no saben salir.

Desde la Psicología se tienen en cuenta unas dimensiones que influyen en nuestras actuaciones. Damasio (2010 apud MARINA, 2012), llama homeostasis al proceso mediante el cual los seres humanos necesitan mantener algunas constantes que se adquieren mediante interacción con el entorno. Sería el mecanismo de "Si pasa esto, haz lo otro". En esto intervienen procesos inconscientes, construyéndose la consciencia sobre estos procesos inconscientes. Una mala instrucción de los procesos inconscientes puede explicar, por ejemplo, por qué algo que nos proponemos no somos capaces de llevarlo adelante, no tenemos suficiente voluntad, por ejemplo en la drogadicción, o al resolver problemas matemáticos, ya que necesitamos instaurar rápidamente la demanda inconsciente de homeostasis.

Otros psicólogos, entre los que se encuentra Angela L. Duckworth y K.Anders Erickson, exponen que cómo nos esforzamos va a tener influencia tanto en los resultados académicos, como en nuestro aprendizaje. Duckworth y Seligman (2005) ponen de manifiesto que los resultados académicos dependen más de la autodisciplina que de su cociente intelectual, y Erickson (2006) establece que la piedra angular de toda destreza máxima no es 
la genialidad, sino la práctica intencionada: la cantidad de tiempo y energía que uno dedica practicando de forma deliberada, es decir, practicando de forma que vaya más allá de sus capacidades. Otro autor que establece cómo la forma de esforzarnos interviene en los progresos de los alumnos es Pleux (2004), quien en palabras de Marina (2012) establece que la intolerancia a la molestia o al fracaso hace más vulnerable a los alumnos.

Consideramos que al esforzarnos están implicadas creencias, actitudes, emociones y valores, con lo que nuestro trabajo podría enmarcarse dentro del concepto de Dominio Afectivo en el sentido de McLeod (1992), pero este marco no incluye las variables que definen la forma de esforzarnos, como son: la atención, el habla interior (cómo nos hablamos a nosotros mismos) y el sentido que le da el alumno al reto. Así, los objetivos que nos marcamos para este artículo son:

- presentar un marco teórico que incluya las dimensiones atención, habla interior y sentido del reto, en la que las emociones observadas nos servirán para apoyar las actitudes observadas,

- analizar en el modo de atender de la alumna a los problemas, y para ello nos fijaremos en si abandona el problema ante la dificultad y si es capaz de percibir algo que vaya más allá del enunciado,

- indagar cómo es el habla interior de una alumna, por ejemplo si este habla interior le incita a hacer conjeturas, o a comprobarlas,

- analizar si para la alumna los problemas son un reto, qué emociones desencadenan como la inseguridad por dudas, y cómo influye en el proceso de resolución,

- interpretar cómo se esfuerza una alumna, que hemos llamado Sísifo, con el marco anterior.

El artículo tendrá los siguientes apartados: marco teórico en el que se especifican las dimensiones que analizamos, metodología usada, el análisis y los resultados de los datos obtenidos de la participante Sísifo, y las conclusiones y futuros estudios.

\section{Marco teórico}

Supongamos, como hemos dicho antes, que a los alumnos se les ha instruido un modelo de resolución de problemas cuya primera fase es la comprensión, que incluye tener presente las condiciones o informaciones relevantes del problema o lo que debemos obtener. Nuestra experiencia como profesor nos hace ver que hay alumnos a los que se les olvidan las condiciones o informaciones, con lo cual es posible que no comprueben, o que hay alumnos 
que cuando están buscando la solución no tienen en cuenta qué es lo que le piden. Parece que su atención no es todo lo eficaz que necesitan y la planificación y organización de las metas no es útil. Estas habilidades, junto con otras, algunos autores como Marina (2012) ${ }^{1}$, las llaman habilidades ejecutivas. Otros autores como Damasio (2010), estudiando a pacientes con alto cociente intelectual que no son capaces de mantener ciertas tareas o su planificación, consideran también estas habilidades.

Una de las habilidades ejecutivas es dirigir la atención. Para psicólogos como John Bargh (2005), parafraseado por Marina (2012, p. 67), "el papel principal de la consciencia - y por tanto también de la atención - es integrar en una tarea múltiples mecanismos mentales automáticos que pueden funcionar de manera separada, como la percepción, la memoria y las emociones". El modo atento de resolver problemas matemáticos es, en primer lugar, aquel en el que acompañamos conscientemente todas nuestras actuaciones, de tal forma que la aplicación de recursos intelectuales y afectivos es tan grande que no deja espacio mental para la realización de otras operaciones, y, en segundo lugar, es una atención voluntaria dirigida por nuestro proyecto de resolver el problema.

Así, nos fijaremos en cómo la alumna dirige su atención, ¿Deja el problema ante la primera dificultad y su correspondiente frustración?, ¿Intenta reconducir esta frustración? ¿Es capaz de percibir algo que va más allá del enunciado del problema? ¿Tiene presente tanto las condiciones del enunciado y lo que le piden, o tiende a olvidarlas?

$\mathrm{Al}$ resolver problemas, los alumnos se hablan a sí mismo de una forma potenciadora o no. Mientras que unos a la primera dificultad se pueden decir que son tontos, que no les va a salir, que no entienden nada, otros son capaces de lanzarse a hacer conjeturas aunque no estén seguros, o son críticos consigo mismo, comprobando la solución, y otros son capaces de enmarcarlos en un contexto de aprendizaje y ven sentido al fracaso y al éxito para su mejora como resoluto res. Esto es el habla interior del alumno, lo que se dice a sí mismo al resolver problemas. Desde la Psicología, autores como Miller, Galanter y Pribam (1960), parafraseados en Marina (2004), consideran que el habla interior es el material en el que sustentan nuestras actitudes, es decir, existe un lenguaje retórico capaz de movilizar emociones. Marina (2012) destaca la forma en la que nos narramos nuestra propia vida puede ser destructiva o paralizadora, por ejemplo cuando nos decimos que somos unos inútiles: si un

\footnotetext{
${ }^{1}$ Marina (2012) considera habilidades ejecutivas las siguientes: inhibir la respuesta, dirigir la atención, control emocional, planificación y organización de metas, inicio y mantenimiento de la acción, flexibilidad, manejo de la memoria de trabajo y manejo de la metacognición.
} 
alumno continuamente se dice que es un inútil en problemas de Matemáticas estará condicionando su actuación.

También, desde la Didáctica de la Matemática se ha estudiado y se han hecho propuestas en este sentido, como Gil, Blanco y Guerrero (2005), quienes proponen un modelo de 4 fases para resolver problemas, inspirado en el de Polya (1992), cada una de las cuales lleva asociadas autoinstrucciones, frases para decirse uno a sí mismo. Así, la fase Analizar y comprender el problema se corresponde con las autoinstrucciones antes del suceso (fase de preparación), la fase de Buscar una estrategia de solución, con las autoinstrucciones al comienzo del suceso (fase de confrontación), la fase de llevar a cabo un plan y examen, con las autoinstrucciones durante la tarea (fase de afrontamiento), y la fase de revisión de la solución y del proceso, con la fase de reforzamiento del éxito. Schoenfeld (1992, p. 43) propone un habla interior retórico y potenciador, a través de preguntas durante el proceso de resolución: “¿Qué estás haciendo? (¿Puedes describirlo con precisión?),¿Para qué lo estoy haciendo? (¿Cómo encaja en la solución?) y ¿Cómo me ayuda? ¿Qué harás con los resultados que obtengas?)".

Nosotros consideramos que, además de lo que propone Schoenfeld (1992), forman parte del habla retórico potenciador el decirse frases como estas: me lanzo a hacer conjeturas, aunque no esté seguro y ¿Seguro que va bien?

Aunque escapa de las metas de nuestro artículo la definición de problema, podríamos decir que cualquier situación planteada con finalidad educativa que propone una cuestión matemática se acerca más a un problema, cuanto más requiera el uso de las habilidades ejecutivas. Como vemos con esta definición de problema, podemos caracterizar un problema, no siendo necesario mencionar el aspecto subjetivo de esta definición, ya que la subjetividad está en las habilidades ejecutivas. Además, este marco teórico intenta integrar la inteligencia vista desde un punto cognitivo, y la inteligencia emocional. Así que, para que haya un problema este debe tener dificultades, que pueden convertirse en un reto o no.

La idea de reto ha sido tratada desde la Didáctica de las Matemáticas por autores como Jaworski y Potari (2002), quienes usan el constructo de triada de la enseñanza como herramienta útil para analizar las complejidades del proceso de enseñanza. Este constructo es el sistema formado por gestión del aprendizaje, sensibilidad hacia el aprendizaje de los estudiantes y reto matemático. El reto matemático se refiere a los desafíos que se le propone al estudiante para que haya pensamiento matemático y actividad, lo cual incluye las tareas, las cuestiones propuestas y el énfasis en cuestiones metacognitivas. Para nosotros, una tarea cognitiva con una dificultad pasa a ser en una persona un reto si la tarea cognitiva provoca 
una emoción de aceptación, que empieza con la intención de resolverlo y acaba, este sentimiento, cuando se resuelve la tarea.

Esto quería decir que en la motivación para resolverla, el incentivo, está el querer hacerlo por nosotros mismos. Así, un problema puede ser un reto para un alumno, pero no para otro, aunque para los dos presente dificultad. Ante este reto, puede que se desencadenen emociones como felicidad, tristeza, miedo, ansiedad, disgusto e interés, u otras más complejas basadas sobre estas, como propone Hannula (2002). Gómez-Chacón (2003) desglosa las emociones negativas que pueden desencadenarse como ansiedad, miedo, temor, la desesperación, el desconcierto o inseguridad, comerse la cabeza, bloqueo y frustración. Todas ellas podríamos unificarlas bajo el paraguas de sufrimiento, y consideramos interesante esta unificación para destacar este concepto inherente y presente siempre en el ser humano.

Algunas diferencias con respecto a otros marcos, como el de Hannula (2002) o Gómez-Chacón (2003) son que las emociones son elementos que nos ayudarán en el análisis que hacemos, y no la dimensión fundamental que analizar, y en segundo lugar que al centrarnos en cómo atienden, cómo es su habla interior o cómo enfocan el reto, nos basamos en lo que manifiestan que creen (posiblemente creencias), lo que hacen y las emociones que ellos sienten, pero a estas no las calificamos siempre como emociones positivas o emociones negativas $^{2}$.

\section{Metodología}

Esta investigación es parte de una investigación más amplia, que trata sobre el esfuerzo en ambientes de Resolución de Problemas matemáticos, tanto con alumnos como profesores. El estudio con alumnos se llevó a cabo con alumnos de $3^{\circ}$ y $4^{\circ}$ de ESO del primer autor, que actuó como profesor-investigador.

Durante las clases de $3^{\circ}$ de ESO los alumnos conocieron un modelo de Resolución de problemas, que era una adaptación del de Mason, Burton y Stacey (1992), De Guzmán (1991) y Schoenfeld (1985), en el que había heurísticos para la comprensión o familiarización con el problema, heurísticos para buscar un plan, formular conjeturas y la comprobación de estas. Además, en las clases había una fuerte componente de la Resolución de Problemas como metodología en el sentido de Hatfield (1978). Durante el curso siguiente, en $4^{\circ}$ de ESO, la

\footnotetext{
${ }^{2}$ Que nosotros intentemos no calificarlas siempre como positivas o negativas, no quiere decir que el alumno no las viva como positivas o negativas.
} 
Resolución de Problemas seguía sustentándose tanto como contenido como metodología, y se tomaron los datos.

Debido a nuestro interés en profundizar en las realidades de los sujetos participantes en la investigación, decidimos abordar un estudio de casos, dentro del paradigma interpretativo, lo que nos permitió interpretar la riqueza de los datos que obtuvimos. La metodología que usamos fue la etnográfica, en un principio desembocando en un estudio de tres casos tras el siguiente proceso: los 3 alumnos participantes, Sísifo y dos más, durante el curso 2008/2009 cursaban $4^{\circ}$ de ESO en un Instituto de Educación Secundaria de un barrio de la ciudad de Málaga (España). Nos decidimos por un estudio etnográfico al encontrarnos inmersos en el complejo escenario de una clase por ser profesor de estos alumnos, en el que intentábamos describir sus creencias y prácticas. En este escenario que es su hábitat natural (el aula), los alumnos se enfrentaban a los problemas del libro de texto y a exámenes.

En un proceso inductivo, partimos de aquí como para obtener perfiles, teniendo en cuenta el punto de vista de los participantes para interpretar los significados. Tras los primeros datos de los casos, fuimos configurando perfiles, y nos interesó la descripción particular de un tipo de alumno, como Sísifo, intentando que esta descripción fuera rica y lo más profunda posible para comprender qué cree, piensa y cómo actúa. El objetivo principal no es el caso en sí, Sísifo, sino aportar luz sobre cómo se esfuerza, por lo que es un estudio de casos instrumental en el sentido de Stake (2000).

Para la selección de los casos se procedió del siguiente modo. Los alumnos de la clase de $4^{\circ}$ de ESO realizaron el cuestionario. Un análisis de sus perfiles nos llevó a seleccionar a 6 alumnos. La entrevista sobre el cuestionario condujo a los tres alumnos elegidos por ser buenos informantes.

Describimos brevemente cómo era Sísifo. Sísifo era una chica que en las sesiones de evaluación los profesores calificaban como que le costaba bastante; de hecho, había aprobado Matemáticas en Septiembre de $3^{\circ}$ de ESO. Sus notas en Matemáticas en los cursos anteriores solían ser Suficiente. Uno de los comentarios que nos hizo en una de las entrevistas es que en los cursos anteriores no se enteraba de nada de los problemas, y de hecho, en los problemas de los exámenes apreciábamos que ante la primera dificultad no era capaz de buscar métodos alternativos para resolverlos. Por todo lo anterior, por ser buena informante y porque había bastante empatía con nosotros le pedimos que fuera informante.

Los instrumentos de recogida de información son un cuestionario donde aparecen las dimensiones que hemos tratado, entrevistas sobre el cuestionario, la realización de 4 problemas con sus correspondientes entrevistas, problemas que se incluyen en el Análisis. Los 
cuestionarios tienen preguntas con escala de valoración y preguntas abiertas, predominando estas últimas. Las preguntas se analizan en varias categorías, que denominamos sentido de las actividades, sentido de actitudes y valores y sentido de la evaluación, situándose la mayoría de preguntas sobre la atención, reto y habla interior en la categoría de sentido de actitudes y valores. Las respuestas se analizan con un instrumento que consistía en una tabla que valoraba si su atención era plena o no, si su habla interior era retórica potenciadora o no, y si el reto era fuente de aprendizaje y disfrute o no. En los días previos a los exámenes, los alumnos podían preguntar dudas de los problemas propuestos para casa, problemas que no se corregían en clase y que se proponían como mínimo 10 días antes del examen, así que nos pareció que eran las clases idóneas para tomar notas de campo. En las entrevistas queríamos que la alumna profundizara y justificara las respuestas de los cuestionarios, así como las dudas que nos surgían de los cuestionarios o de los protocolos de los problemas. Las dimensiones que nosotros analizamos las separamos por una cuestión operativa, aunque en los instrumentos de recogida de información aparecen conectadas.

\section{Análisis y resultados}

Como hemos comentado, Sísifo era una alumna que en las sesiones de evaluación los profesores de todas las asignaturas la calificaban como una alumna que le costaba hacerse con la materia. Concretamente, cuando se refería a los problemas de matemáticas, decía que hasta $2^{\circ}$ de ESO (un año antes de que conociera el modelo de Resolución de Problemas), no [se] enteraba de nada.

Hemos dividido la narración que hacemos de Sísifo en dos actos: tras pasarle el cuestionario y hacerle una entrevista sobre él, y tras hacer las entrevistas correspondientes a los protocolos de 4 problemas. En las entrevistas, hemos expresado en cursiva los puntos que deseamos destacar en lo que decía Sísifo, y hemos nombrado al entrevistador por $\mathrm{P}$ ( $\mathrm{P}=$ profesor), mientras que los puntos suspensivos indican texto omitido o incisos del entrevistador en las respuestas de la alumna.

\subsection{Análisis de los resultados tras el cuestionario y entrevista correspondiente}

En la entrevista sobre el cuestionario empezamos preguntándole cómo le va en Matemáticas. 


\section{$P:$ ¿Cómo te va en Matemáticas?}

Sísifo: matemáticas sé que me va a costar... lo que según ella le falla sobre todo es a plantearme el problema, porque no sé planteármelo, tampoco sé plantearme el problema ni cómo empezar ni cómo seguir.

Parece que nos está hablando, aquí, de una percepción que no va mucho más allá del enunciado, lo cual nos informaría de su modo de atención. También nos habla de su modo de atender la siguiente parte de la entrevista, aunque también sobre las dificultades asociadas a los retos.

\section{$P: ¿ Q u e ́$ piensas o sientes cuando hay dificultades en un problema?}

Sísifo: cuando estoy leyendo un problema, por ejemplo, y sé que no sé hacerlo, lo que estoy leyendo no lo entiendo es cómo que vuelvo a leerlo unas cuantas veces y es igual, me quedo igual, o sea me agobio, ya me agobio mucho, y ya como si lo dejase, pues voy a hacer esto a ver si estuviera bien, lo dejo incluso sé que está mal, yo sé que está mal...

Parece indicarnos falta de una percepción más allá del texto del enunciado, así como su falta de capacidad para gestionar la falta de comprensión significativa.

Cuando le preguntamos abiertamente sobre su habla interior, no parece informarnos sobre si ésta es retórica potenciadora o no, y se aprecia cierta motivación por querer resolver el problema y no abandonarlo.

Sísifo: que cómo puedo tirar, cómo puedo solucionarlo y o sea primero me agobio mucho yo ya ahí lo dejo, porque cuando me agobio, o sea no es que lo deje, lo que no sé lo dejo para el final hago lo que sé y vuelvo a estar con lo que no sé y ahora leyéndolo, intentando hacerlo, tanteando el problema y eso.

Como vemos, no contesta mucho sobre su habla interior, así que es probable que haya ausencia de ella.

En los comentarios anteriores se entremezclan informaciones sobre su idea de reto, y, a continuación, exponemos cuando le preguntamos qué le parecía que en las clases de Matemáticas hubiera retos, es decir, problemas:

Sísifo: sí quizás, no sé, así sé a donde tengo que llegar, es como que motiva más, motivarme para llegar al final y hacerlo, aunque lo mismo me puede costar un montón hacerlo y al final no llegar ni nada... que por ejemplo si usted me dice que tengo que llegar a una solución como un reto o lo que sea, pues como que me motiva más llegar a la solución porque sé que tengo que llegar, como si me da más fuerza a lo mejor quizás el saber que tengo un reto y como yo sé que quiero hacerlo ... 
Esta respuesta parecía contradecir lo que observamos en los días de dudas previos a los exámenes, en los que se dedicaba a hacer los ejercicios no corregidos, pero no sobre los problemas, así que decidimos volverle a hacer la pregunta de otra forma:

P: Sin embargo hay alumnos que ni preparan los problemas de casa, ni pregunta dudas en los días previos al examen. ¿Cómo ves tú eso?

Sísifo: a mí también me ha pasado ... los problemas de casa dificiles yo me imagino que usted no los va a poner ... y los suelo dejar.

Aunque puede que haya cierto ánimo para involucrarse en el reto, incluso en los que tiene bastante tiempo para resolverlos, ella se evade de ellos. Volvimos a hablar del reto cuando le preguntamos si ella estaba sufriendo en las clases de Matemáticas.

Sísifo: sí, sí tampoco sufriendo pero sí me preocupo por la asignatura ... bastante agobiada, sé que hago el examen y además yo hago el examen y sé que me ha salido mal y voy a suspender, tampoco es que me frustro, siempre saco un 3 o incluso menos de un 4, 3, 3.5 o 4 y como que de ahí no paso, yo veo que no apruebo, que no apruebo y es que me agobio un montón, me frustro.

Aunque no estaba consensuado entre entrevistador y entrevistado qué entendíamos por sufrimiento, parece que ella lo interpreta como frustración y su correspondiente preocupación.

Nuestra interpretación de las informaciones que amablemente nos dio Sísifo es que su atención no era muy plena a la hora de resolver problemas, acompañada de una emoción como la frustración. Esta frustración no se gestionaba de forma que sirviera para avanzar en la resolución del problema, y como prueba de ello está la probable ausencia de un habla interior potenciador. También el reto se relacionaba con la frustración, que le hacía abandonar la tarea aunque tuviera tiempo para resolverla.

\subsection{Análisis de los problemas}

Para la elección de los problemas les impusimos las siguientes condiciones: su adecuación a la definición de problema, al contexto y a que tuvieran potencial heurístico. Que se adapte a la definición de problema requiere que se preste al uso de las funciones ejecutivas, como dirigir la atención, planificar las metas que te propone el problema u otras intermedias, hacer uso de la memoria para acceder a procedimientos, conceptos o estrategias ya usadas o manejar la metacognición en el sentido de Schoenfeld (1992). Tener en cuenta el contexto implica que su solución o métodos de solución incidan en estructuras conceptuales aconsejadas en el currículum de $4^{\circ}$ de ESO y en hacer más flexible el esquema de Resolución 
de Problemas. Así que tenga en cuenta el contexto implica que se presten a conjeturar, comprobar y justificar las conjeturas. En cuanto al potencial heurístico, lo entendemos como que se presten a usar los heurísticos, contextualizados o no, que el alumno ya ha usado.

Los problemas se presentaron como sigue:

- Les entregamos el enunciado del problema a toda la clase de $4^{\circ}$ ESO en la que está Sísifo, de forma que no podían comentar nada sobre su proceso. Se les dijo que se les valoraría como una nota de clase y tendrían aproximadamente 50 minutos para su resolución.

- Analizamos lo que realmente ha escrito en papel el alumno, intentando identificar fases y atascos.

- Entrevistamos al alumno entre 10 y 20 minutos después de la resolución en clase. La entrevista se haría en función de su proceso de resolución en papel, y de las fases y atascos, o de alguna contingencia que nos sugiera el entrevistado, para ver si nos da información de los datos que quedan por confirmar tras el cuestionario y la entrevista.

- Si pasa mucho tiempo atascado le dimos una ayuda mínima.

\subsubsection{El problema 1 y su análisis}

Este problema tenía por título La Flauta Mágica y su enunciado fue:

La Flauta Mágica ${ }^{3}$ : Gumersindo Peláez tiene una flauta que mide $26.8 \mathrm{~cm}$, que quiere guardar en una caja de zapatos con dimensiones 10, 15 y $20 \mathrm{~cm}$. Él dice que sí le cabe, mientras que su amigo Antoñito afirma que no. ¿Cuál de los dos tiene razón? Generaliza con una fórmula el resultado del apartado anterior.

Su esquema de resolución fue muy pobre, haciendo el dibujo de un rectángulo en un primer momento, pero como se llevó un tiempo atascada, le sugerimos que hiciera una caja en 3D, a la que acompañó de una flauta en una pared. Calculó la diagonal de esta pared de $15 \mathrm{x}$ 20 con el teorema de Pitágoras, obteniendo $25 \mathrm{~cm}$, y afirmando que la flauta no cabía y que quizás sí si la pusiera inclinada. Se notaba en el papel que hizo esto después de borrar el cálculo de la diagonal de la pared de 10 × 20.

Le preguntamos si le había parecido difícil el problema, contestando:

Sísifo: fácil... porque me esperaba a lo mejor un problema más difícil y he sabido por donde he tenido que ir y tal, o sea que bien vamos ... porque leyendo el problema

\footnotetext{
${ }^{3}$ Este problema se presentó cuando se estaba dando la Unidad Didáctica de Números Reales, en la que apareció y se repasó el Teorema de Pitágoras. Se había comentado, también, el heurístico contextualizado siguiente: cuando te pidan una distancia busca un problema análogo, como por ejemplo con Teorema de Pitágoras.
} 
sabía de qué es lo que iba y que es lo que tenía que hacer respecto a la fórmula y tal, porque en otras ocasiones leo el problema y no sé por donde tengo que empezar.

Nos pareció que el entender algo y el saber lo que tiene que hacer la ha animado. Como borró el cálculo de una diagonal que le podía haber ayudado, le preguntamos por si había tenido dudas, y con su respuesta pudimos interpretar datos de su atención y cómo afrontaba el reto cuando ella cree que no lo va resolviendo:

Sísifo: lo que tenía dudas es a ver si seguía bien y estaba bien lo que estaba haciendo $y$ de a lo mejor quizás como podía colocar la caja y de qué manera colocar la flauta para que valiese.

En cuanto a su habla interior nos dijo.

Sísifo: yo lo que pensaba es cómo podía seguir, qué podía hacer porque a lo mejor escuchaba y decía estoy haciendo lo que tienen otros... a ver si estoy haciendo cosas que no sirven para nada.

Este habla interior no ha activado que compruebe.

Desde nuestro marco teórico, interpretamos que no hubo atención plena que le ayudara, como prueba el hecho de la falta de percepción de hacer el dibujo en 3D, o que no ha sabido gestionar las dudas. Estas dudas y la falta de un habla interior más potenciador para gestionarlo, impidieron que el reto continuara con una comprobación. El ánimo del principio por entender algo no tuvo suficientes frutos, porque la inseguridad le impidió la persecución de la eficacia, que comienza con una comprobación.

\subsubsection{El problema 2 y su análisis}

El problema 2 tenía por título Cuadrados y Cerillas II y su enunciado fue este:

Cuadrados y Cerillas II': ¿Cuántas cerillas se necesitan para construir $n^{2}$ cuadrados de lado 1 formando otro cuadrado mayor, como en la siguiente sucesión?

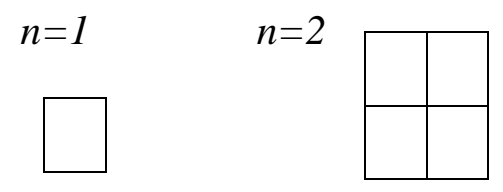

Ella continuó con los cuadrados para $n=3$ y $n=4$, que hizo correctamente. Su esquema de resolución volvió a ser pobre, sólo usando la estrategia de la particularización, aunque sin aprovechar suficientemente estos tanteos. Obtuvo una fórmula $\mathrm{y}=4 \mathrm{n}$ para las diferencias, que

\footnotetext{
${ }^{4}$ Este problema aparecía en los apuntes de Álgebra que tenían los alumnos y que complementaban al libro de texto. Se había trabajado ya uno más fácil llamado Cuadrado y Cerillas I, con el que se trabajaron los conceptos de variable, fórmula e identidad, y el atreverse a hacer conjeturas y comprobar para la obtención de fórmulas.
} 
no explotó para su cometido. Al final, le dimos una ayuda de que el caso $n=3$ necesita $3 \cdot 4+4 \cdot 3$ cerillas, que no aprovechó.

No llegó a percibir otra forma de ver los cuadrados como prueba el hecho de que no explotó la ayuda de $3 \cdot 4+4 \cdot 3$. En cuanto a la atención plena, empezó con ánimo pues vio que es algo parecido a un problema anterior, pero la dificultad y su frustración le hizo dejarlo.

P: Con la fórmula $y=4 n$ parece que te quedaste atascada.

Sísifo: Un, al saber esa fórmula yo sabía las diferencias pero me faltaba la que realmente valía, la del número de cerillas de cada cuadrado y eso yo ya no sabía hacerlo, y yo creo que me hubiese costado bastante llegar a la solución ... y ya me agobié y empezaba a dejarlo, no sabía qué relación había entre la fórmula y la que tenía que hacer.

Creímos que también nos informaba de que la dificultad en el reto desembocaba en inseguridad. También le preguntamos qué si este reto le hizo estar más confiada que en el problema anterior:

Sísifo: sí ... pero no es por nada, porque al hacerlo yo sola, cuando usted me ha puesto atrás yo sola, bueno pues entonces yo en el examen estoy yo más suelta, si estoy yo sola y sé que puedo hacerlo me suelto más...

Así, nos pareció que el reto de hacerlo por sí misma la anima, en un principio, la animó a involucrarse.

En cuanto a su habla interior, le volvimos a preguntar qué se decía mientras resolvía el problema:

Sísifo: yo pensando para mi diciendo si veo que puedo sacar adelante el problema, este y el otro también pues a la hora de hacer el examen, seguramente pueda hacer, no es por nada, si voy practicando haciéndolo sola y tal al final siempre me suelto más y a la hora de hacer el examen lo haré mejor yo sola.

Vimos que es un lenguaje muy vago e impreciso para activar su memoria.

La interpretación que hicimos fue parecida a la del problema anterior. El que haya cierta familiaridad con otro problema le hizo entender algo, y la animó, aunque no con la suficiente paciencia para percibir aspectos del problema, o para tener una atención más plena. Esta atención empezó a desvanecerse cuando el reto presentó una dificultad que le creó dudas e inseguridad, que podría completarse con una comprobación. Su habla interior no fue potenciador hacia la eficacia. 


\subsubsection{El problema 3 y su análisis}

El tercer problema que les planteamos fue Buscando Refugios Desesperadamente, con el siguiente enunciado:

Buscando Refugio Desesperadamente ${ }^{5}$ : este es un desierto rectangular. A, B y C son las entradas de tres refugios antinucleares. Colorea de diferente color las zonas desde las que te dirigirías a cada refugio en caso de alarma nuclear.

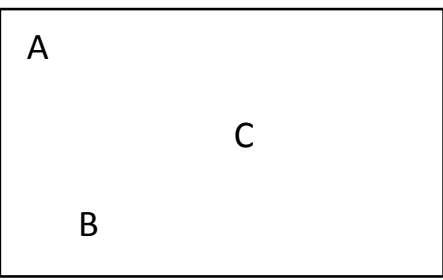

En el tercer problema hicimos una variante a la metodología. Observamos en el último problema que quizás los alumnos no ponían suficiente motivación para ser eficientes en la resolución, e incluso, todo el interés que parecían mostrar en los días de dudas no se reflejaba en la resolución de los problemas. Por ello la variante que introdujimos fue la siguiente:

- les invitamos, tras terminar una unidad didáctica y en una fecha cercana al examen, a estudiar en la Biblioteca del Instituto. Los observaríamos y participaríamos lo mínimo, y tomaríamos notas de campo sobre lo que hacían los alumnos, nos preguntaban o hablaban entre sí,

- los alumnos, en esta época, ya debían estar intentando resolver los problemas de casa para el examen, y les dijimos que pueden comunicarse tal y como lo harían en sus casas,

- al terminar esta sesión con los alumnos, les invitaríamos a cenar con la intención de no dejarles tiempo para que en casa estudien más matemáticas. En esta cena no se hablaría de las clases de matemáticas, y al día siguiente a primera de hora de la mañana, sin previo aviso, les pusimos el Problema 3 (de Geometría clásica), analizamos el protocolo y los entrevistamos sobre el mismo.

En las notas de campo, primero hizo un dibujo como el tercero del protocolo, y le ayudamos diciéndole que lo hiciera más fácil con dos refugios. A los 5 minutos como no reaccionaba con la ayuda que le dimos, le sugerimos que empezara por dos refugios, y a los

\footnotetext{
${ }^{5}$ Este problema estaba propuesto para casa en la Unidad Didáctica de Lugares Geométricos. Se había hecho en clase uno parecido titulado Gasolinera entre dos pueblos, en el que se pedía dónde situar una gasolinera entre dos pueblos de forma que equidistara de ellos. Con el se trabajó el concepto de mediatriz y la particularización organizada como heurístico.
} 
10 minutos de la segunda ayuda nos volvió a enseñar el dibujo con tres refugios, y le volvimos a decir que se olvide del tercer refugio. Aún así no avanzó.

Al día siguiente le pusimos el mismo problema en clase, y el protocolo de Sísifo fue el siguiente:

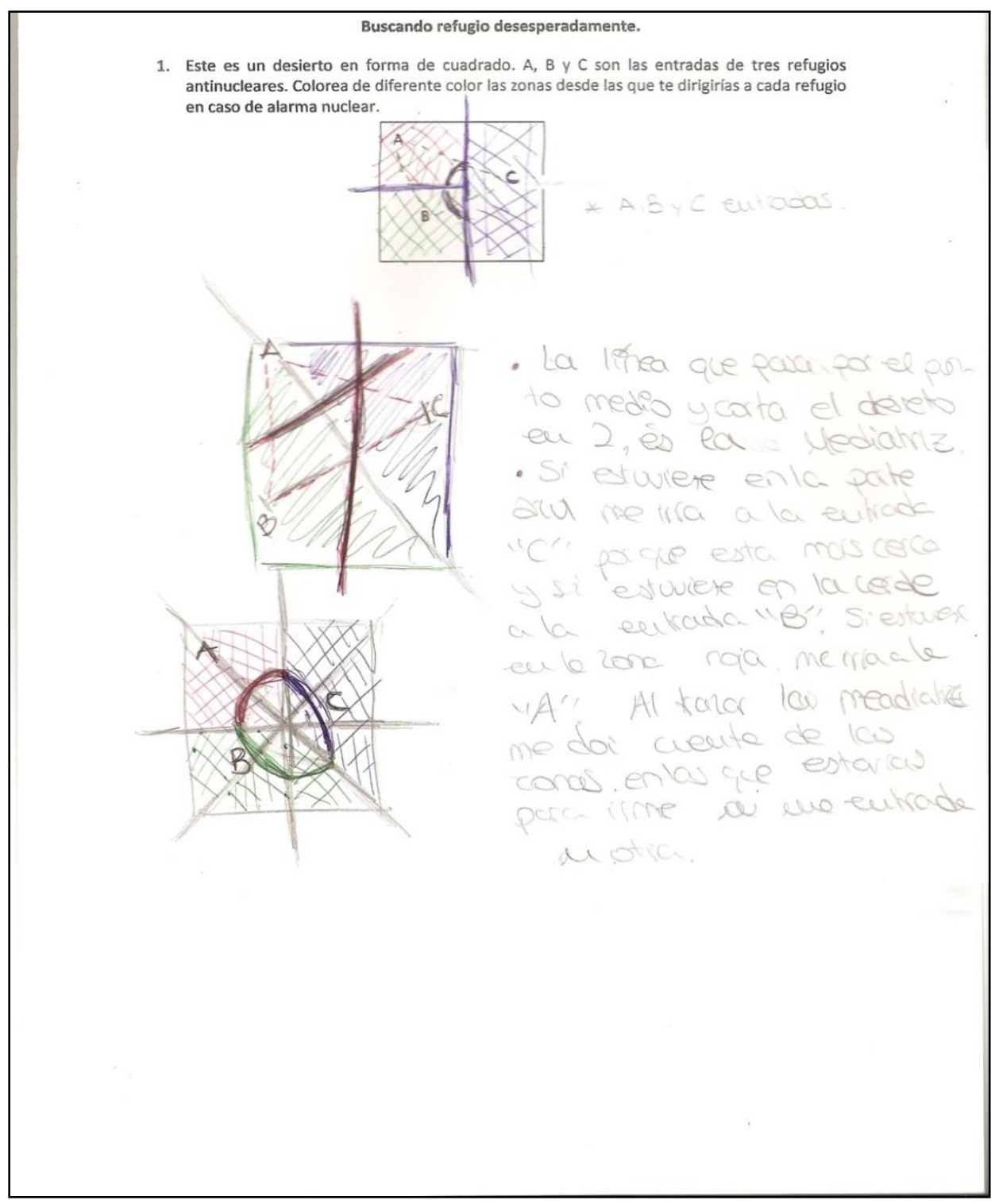

Figura 1 - Protocolo de la alumna en el problema 3

Fuente: datos de la investigación

En la entrevista le preguntamos por el dibujo $2^{\circ}$ del proceso en el que las mediatrices no están correctamente representadas, y no ha comprobado si es correcto lo que expresa con colores (hay tres zonas coloreadas cada uno expresando el refugio al que escaparía)

Sísifo: ... sí me he dado cuenta pero no lo he dejado pasar ... Y lo he dejado, ya veía yo mucho lío, bueno voy a dejarlo así ... y ... Lo dejo así. Si tengo claro que puedo 
hacer lo siguiente, lo borro y lo hago de nuevo y lo hago, si no tengo claro lo que voy a hacer lo dejo así y ya cuando se corrija lo verét.

La información que aportó este problema no variaba mucho de los anteriores, con lo que pensamos que la variante metodológica introducida no aportó mucho.

Como vemos su atención estuvo muy pendiente de su inseguridad, y en el reto no había sido tal, solo había sido un reto al principio, pues la emoción de querer resolverlo acabó cuando la inseguridad cobró más fuerza, impidiendo que comprobara.

\subsubsection{El problema 4 y su análisis}

A pesar del cambio metodológico introducido, seguíamos pensando que los alumnos participantes en la muestra no ponían todo el interés deseado al resolver los problemas que les planteábamos para este estudio. Así, para el problema 4, decidimos hacer otro cambio en la metodología:

- $\quad$ observamos y tomamos notas de campo de los dos días de dudas de la Unidad Didáctica (sería la semana siguiente a la cena). Sería de trigonometría,

- al día siguiente uno de los problemas que era parecido a los propuestos para casa, se lo pusimos como primer problema del examen. El enunciado de este problema figuraría solo en el folio, y les dimos 45 minutos para resolverlo. Tras estos 45 minutos con este problema, les pasamos el resto de preguntas para las que dejamos 60 minutos,

- hicimos una entrevista sobre este primer problema, tras los 90 minutos de examen, que se correspondería con el Problema 4 que usamos como recogida de información.

El problema 4 fue el siguiente:

Examen de trigonometría de $4^{{ }^{7}}$ : Los dos ángulos de un triángulo rectángulo se llaman complementarios porque su suma es $90^{\circ}$. ¿Cuál es el complementario de $10^{\circ}$ ¿Qué relación hay entre la tangente de un ángulo y la tangente del complementario?

Según consta en las notas de campo, Sísifo no nos preguntó sobre si este problema era parecido al mandado para casa. Dibujó un triángulo rectángulo con un ángulo de $10^{\circ}$, y a

\footnotetext{
${ }^{6}$ Hay que aclarar que en las notas de campo de que vinieron a pedirme ayuda en los días de dudas, me comentó que nunca comprueba porque prefiere no sentirse insegura por si está mal.

${ }^{7}$ Este problema se puso al principio del Examen de Trigonometría. Para casa había mandado uno parecido a éste, en el que se pedía qué relación hay entre el seno de un ángulo y el coseno del complementario y que, como todo problema de casa, no se había corregido.
} 
continuación calculó el seno y el coseno de $45^{\circ}$, escribiendo la conjetura: el seno y el coseno salen lo mismo. A continuación puso tres ángulos y sus complementarios respectivos, y dividió el seno de un ángulo entre el coseno del complementario, que como le salió siempre 1 llegó a la conclusión de que la relación entre la tangente de un ángulo y la tangente del complementario es 1 .

Cuando le preguntamos cómo le había ido en el problema nos dijo:

Sísifo: me resulta fácil por haberlo hecho antes, ... tuve dudas hasta que saqué la conjetura e hice lo que creí que era...calcular la tangente, bueno seno entre coseno las calculaciones que había hecho antes y lo hice del ángulo, hice el complementario y luego me salió.

Como vemos, estuvo lejos de perseguir la eficacia. Parecía que no hay una atención muy plena, pues ni siquiera percibió qué es lo que le piden, por no pararse suficiente tiempo en la comprensión del problema, esto último a pesar de que ella creyó que sí lo ha comprendido cuando le preguntamos si había comprendido bien el problema:

Sísifo: sí porque al principio yo pensaba, mira si soy tonta, que al leer el problema eeeh, pensaba que era igual y ni siquiera me había leído bien las preguntas... y entonces al leer las preguntas me he... Y entonces ya lo lei y ya creo que lo he comprendido, yo creo que sí, que sí sé lo que me preguntaban.

A continuación le preguntamos si había comprobado, respondiéndonos que:

Sísifo: no he comprobado, como que daba por hecho que así estaba bien, completo o quizás que no he querido comprobarlo por si estaba mal ... y no le quiero dar más vueltas ... si le doy más vueltas y por una cosilla que me he equivocado y por otra no se qué ... estaba segura, pues entonces por eso no le he dado más vueltas.

Otra vez la inseguridad impidió que comprobara, y por tanto que se acercara a ser un reto para ella este problema. La atención estuvo más pendiente de su inseguridad, que de la eficacia. En cuanto al habla interior parecía que sí le hizo ver que el problema no era exactamente igual al mandado para casa, aunque no le ayudó a comprobar.

Empieza habiendo un reto pero se acaba diluyendo, sin que hubiera una atención plena que implicara comprobar por la inseguridad. A diferencia del problema 3, avanzó en la resolución, aunque sin eficacia.

Podríamos resumir que los esquemas de resolución de Sísifo fueron pobres, y que su esfuerzo fue un hacer por hacer, más que una actuación de forma creativa para perseguir la eficacia. Otras formas de esforzarse se podrán ver en otro artículo, con un estudio de casos de una alumna que llamamos Ulises. 
El último problema se realizó en Abril, y durante el tercer trimestre las notas de campo nos decían que la alumna preguntaba más dudas, y que era capaz de resolver algunos problemas mandados para casa con nuestra ayuda. Algo cambió que le hizo mejorar en sus resultados. Prueba de ello es que en la entrevista final, que le hicimos a los alumnos participantes sobre las dimensiones estudiadas, al preguntarle por su habla interior, contesta:

Sísifo: ehh, sí para empezar es que antes llegaba a los exámenes diciendo que voy a suspender y ahora voy con ganas de aprobar, y con más posibilidades de aprobar que de suspender, y así me siento veo el problema y tal y al leer el problema y tal sé lo que tengo que decir, porque me suelo decir voy a leer bien el problema, voy a hacértelo con los folios ... veo los datos, apunto los datos y cuando sé lo que me pide pues voy a empezar a hacer tal y cual, y si me pide un dibujo y ahora a partir de ahí voy a los datos y a hacer lo que hemos hecho en clase ... me digo venga que yo puedo, venga, me digo me da hasta vergüenza suelo decirme, a ver si puede Patri, o puede Claudia o puede Estefanía a ver yo por qué no voy a poder ... y si me atasco empiezo a decir, bueno primero paso a otro ejercicio a ver si me resulta más fácil_y si no empiezo a no sé voy a sacar la conjetura a ver si puedo avanzar más el problema y si me atasco ya es más difícil de sacarlo, porque si me atasco es que ya no sé seguir el problema ...

Ahora, casi al final de curso, hay un habla interior más potenciador, llegando a pensar en heurísticos para resolver problemas o en intentar sacar conjeturas, y su actitud hacia el examen cambió, aunque no parece haber cambios en su habla interior para superar atascos o para comprobar.

La cuestión de qué es lo que ocurrió para que ella cambiara es una pregunta interesante y que queda pendiente para futuros estudios, así como profundizar en el marco teórico que nos ha permitido hacer este análisis.

\section{Conclusiones}

El análisis presentado pone de relieve que este marco teórico nos permite dar forma al perfil de Sísifo. Parece que su atención excesiva a la incertidumbre le hace no comprobar, ya que pudiera estar mal y eso aumentaría su desconcierto. Puede que los problemas empiecen siendo un reto, pero el ánimo por hacerlo se va desvaneciendo por lo anterior. En este proceso el lenguaje retórico potenciador es escaso, de forma que no activa la memoria para acceder a lo que ha practicado antes. Su forma de concebir la inseguridad puede hacer que Sísifo abandone un problema si no lo resuelve en menos de 10 minutos.

Por todo lo anterior podríamos decir que su esfuerzo es más bien un hacer por hacer. Esto nos permite corroborar que las dimensiones atención, habla interior y sentido del reto influyen a la hora de actuar como resolutores, con lo que cumplimos algunos de los objetivos 
que nos planteamos, además de afianzarnos en que el marco teórico elegido es adecuado para estudiar las actuaciones en resolución de problemas. Además, este marco teórico aporta luces sobre conexiones (no todas vistas en este artículo) entre atención, lenguaje y reto, por un lado, y las fases de resolución de un problema, por otro.

Por último, relacionamos los resultados de este artículo con otros resultados sobre resolución de problemas. En Gómez-Chacón (2003), se califican las emociones como positivas o negativas, no siendo este nuestro caso, pues al considerar el reto como inherente a la instrucción y aprendizaje de los alumnos como resolutores, están presentes la dificultad, la incertidumbre y la frustración, que pueden llegar a tener sentido en el aprendizaje.

En el marco de Hannula (2002), se pueden calificar actitudes hacia la matemática como positivas aunque se abandone el problema, caso que no es el de nuestro marco, donde se podría explicar con una mala gestión de la atención, habla interior o sentido del reto.

Nuestro marco se acerca al de Schoenfeld (1992), aunque con diferencias importantes que permiten extraer conclusiones distintas ante actuaciones de los alumnos. Por ejemplo, hemos observado que, si el enunciado tiene algo familiar para el alumno, y esto le permite hacer algo, se lanza a hacerlo sin darse tiempo para una atención más plena, y esto, unido a que la alumna no comprueba porque su sentido del reto no contempla la inseguridad, implica fracasos en las resoluciones. Mientras que Schoenfeld lo explicaría por la creencia de que los alumnos abandonan un problema si tras 10 minutos no lo resuelven, nosotros creemos que la creencia anterior es derivada de la forma en que concibe la atención, el habla interior y el sentido del reto.

Quedan abiertas preguntas como la idea de esfuerzo en los profesores, en padres, de otro tipo de alumnos, sobre el proceso de aprendizaje a largo plazo de los alumnos como resolutores de problemas y sobre la forma de esforzarse de los mismos alumnos en otras asignaturas que conlleven retos.

\section{Referencias}

BARGH, J. Bypassing the will: towards desmytifing the nonconscious control of socila behaviour. In: HASSIN, R. R.; ULEMAN, J. S.; BARGH, J. (Ed.). The new unconscious. New York: Oxford University Press, 2005. p. 37-61.

DAMASIO, A. El error de Descartes. Barcelona: Drakontos Bolsillo, 2010.

DE GUZMÁN, M. Para pensar mejor. Madrid: Labor, 1991. 
DUCKWORTH, A. L.; SELIGMAN, M. E. P. Self-Discipline out'does IQ Predicting Academic Performances of Adolescent. Psychological Science, Thousand Oaks, v. 16, n. 12, p. 939-944, 2005.

ERICKSON, A. The influence of experience and deliberate practice on the development of superior expert performance. In: ERICSSON, K. A.; CHARNESS, N.; FELTOVICH, P. J.; HOFFMAN, R. R. (Ed.). The Cambridge Handbook of expertise and expert performance. Cambridge: Cambridge University Press, 2006. p. 637-705.

GIL, N., BLANCO, L.; GUERRERO, E. El dominio afectivo en el aprendizaje de las Matemáticas. Una revisión de sus descriptores básicos. Unión, Revista Iberoamericana de Educación Matemática, La Laguna, Tenerife, v. único, n. 2, p. 15-32. jun. 2005.

GÓMEZ-CHACÒN, I. M. La tarea intelectual en Matemáticas. Afecto, meta-afecto y los sistemas de creencias. Boletín de la Asociación Matemática Venezolana, Caracas, v. 10, n. 2, p. 225-247, 2003.

HANNULA, M. S. Attitude towards mathematics: emotions, expectations and values. Educational studies in Mathematics, Dordrecht, v. 49, n. 1, p. 25-46, Jan. 2002.

HATFIELD, L. Heuristical emphases in the instruction of mathematical problem solving: rationales research. In: HATFIELD, L.; BRADBARD, D. (Ed.). Mathematical problem solving: papers from research work shop. Columbus, Ohio: Eric/Smeac 1978. p. 21-42.

JAWORSKI, B.; POTARI, D. Tackling complexity in mathematics teaching development: using the teaching triad as a tool for reflection and analysis. Journal of Mathematics Teacher Education, Dordrecht, v. 5, n. 4, p. 351-380, Dec. 2002.

MARINA, J. A. Teoría de la inteligencia creadora. Barcelona: Anagrama, 2004.

MARINA, J. A. La inteligencia ejecutiva. Barcelona: Ariel, 2012.

MCLEOD, D. B. Research on affect in mathematics education: a reconceptualization. In: GROWS, D. (Ed.). Handbook of Research on Mathematics Teaching and Learning. New York: Macmillan, 1992. p. 575-596.

MASON, J.; BURTON, L.; STACEY, K. Pensar Matemáticamente. Madrid: Labor-MEC, 1992.

POLYA, G. ¿Cómo plantear y resolver problemas? Princeton: University Pres, 1992. Traducción Editorial Trillas, México.

PLEUX, D. Manual de educación para el uso de padres. París: Odile Jacob, 2004.

SCHOENFELD, A. H. Mathematical problem solving. New York: Academic Press, 1985.

SCHOENFELD, A. H. Learning to think mathematically: Problem solving, metacognition and sense making in mathematics. In: GROWS, D. (Ed.). Handbook of Research on Mathematics Teaching and Learning. New York: Macmillan, 1992. p. 334-370.

STAKE, R. E. Case Studies. In: DENZIN, N. K.; LINCOLN, Y. (Ed.). Handbook of qualitative research. Sage Publications: Thousand Oaks, 2000. p. 435-454.

VILA, A.; CALLEJO, M. L. Matemáticas para aprender a pensar. Madrid: Narcea, 2004. 